\title{
New planetary nebulae with ISM interaction discovered with IPHAS
}

\author{
Laurence Sabin $^{1}$, Romano L. M. Corradi ${ }^{2}$, Quentin Parker ${ }^{3}$, \\ Antonio Mampaso ${ }^{2}$ and Albert Zijlstra ${ }^{4}$ \\ ${ }^{1}$ Instituto de Astronomía, Universidad Nacional Autónoma de México, Ensenada, B.C, Mexico \\ email: 1sabin@astrosen. unam.mx \\ ${ }^{2}$ Instituto de Astrofísica de Canarias, C/Vía Láctea s/n, 38200 La Laguna, Tenerife, Spain \\ ${ }^{3}$ Department of Physics and Astronomy, Macquarie University, NSW, 2109, Australia \\ ${ }^{4}$ Jodrell Bank Centre for Astrophysics, University of Manchester, Manchester, M13 9PL, UK
}

\begin{abstract}
The low surface brightness usually associated with nebulae mixing with the ISM has long been a substantial obstacle in the observation and statistical study of these interactions. Thanks to the detection capability of the INT Photometric H-alpha Survey (IPHAS), in terms of sensitivity and imaging resolution, we were able to detect and select tens of examples of candidate Planetary Nebulae apparently at different stages of interaction with the interstellar medium (PNe-ISM hereafter) following the Wareing et al. (2007) classification. A spectroscopic investigation was conducted with the San Pedro Martir $2.1 \mathrm{~m}$ telescope (SPM) in Mexico and we present the first results involving a proper classification and analysis of our candidates. The main difficulty is visually separating PNe-ISM from other faint asymmetric nebulosities such as old HII regions, SNRs and general diffuse H-alpha structures. This investigation is a first step in a more comprehensive study of PNe-ISM as more candidates are uncovered by the IPHAS team.
\end{abstract}

Keywords. ISM: general, planetary nebulae: general

\section{Introduction}

Planetary Nebulae (PNe) suffer different physical and morphological changes in the course of their short existence and one of the reasons is their interaction with the surrounding Interstellar Medium (ISM). Indeed, the evolution of the density ratio between both media strongly affect the geometry of PNe which basically evolve from freely expanding nebulae to totally diluted and destroyed shells (when the ISM density overcomes that of the PN). Several theoretical and observational studies have been realised on the subject (e.g. Borkowski et al. 1990; Ali et al. 2000) mainly regarding the earliest (and brightest) stages of interaction. However, the study of such interaction processes at more advanced stages has been made difficult due to the low surface brightness usually associated to interacting $\mathrm{PNe}$ which has prevented their detection in past surveys. Therefore the associated physical processes are also not well known. With the new generations of $\mathrm{H} \alpha$ surveys the detection capability has greatly improved. We therefore present the study of a sample of new PNe discovered in the framework of the IPHAS survey in the Northern Galactic Plane (Drew et al. 2005, Corradi et al. this book, Sabin et al. 2010) which all display an interacting front with the Interstellar Medium. The objects, detected via a systematic visual inspection of $5 \times 5$ arcsec $\mathrm{H} \alpha$ binned mosaics images, were classified according to the scheme by Wareing et al. (2007). The strength of the IPHAS survey mainly resides in the depth reached allowing the detection of extended structures down to $\simeq 10^{-17} \mathrm{erg} / \mathrm{cm}^{2} / \mathrm{s} / \operatorname{arcsec}^{2}$ at full resolution $(0.33 \% /$ pixel) and therefore to target stages from WZO2 to WZO4. 


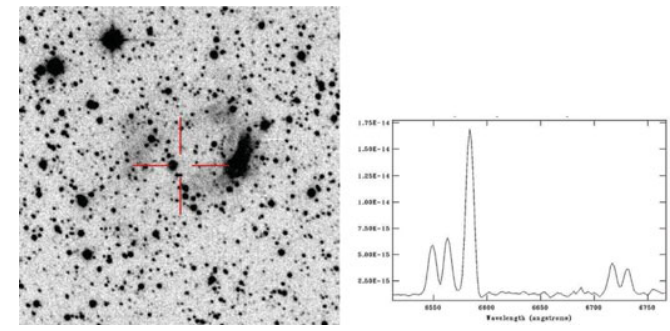

Figure 1. IPHASX J185744.5+105053 is a large WZO3/WZO4 PN (110" size) with a NW direction of motion. We observe a dramatic increase of the [NII] emission probing the interaction process $([\mathrm{NII}]=3.8 \mathrm{H} \alpha)$. A lower limit on the density of about $200 \mathrm{~cm}^{-3}$ was measured and therefore probe the local ISM density.

\section{Spectroscopy Follow-up}

A spectroscopy follow-up was recently realised with the $2.1 \mathrm{~m}$ telescope associated to the low resolution Boller \& Chivens spectrograph at San Pedro Martir Observatory (OANMexico) and the first nine PN-ISM candidates have been investigated. The ratios of the emission lines we were able to detect and measure $(\mathrm{H} \alpha /[\mathrm{NII}]$ and $\mathrm{H} \alpha /[\mathrm{SII}])$ have then been used in the diagnostic diagrams by Frew and Parker (2010). As a result, out of 9 candidates 7 are found to be true PNe (e.g. Fig. 1) while the remaining two are more likely to be related to HII regions. Our results indicate a seemingly correlation between the different stages of interaction and the $[\mathrm{NII}]$ and $[\mathrm{SII}]$ emission in the PNe. Thus, the more advanced evolutionary status is found at the bottom left of the diagnostic diagram where the nitrogen and sulphur intensities supersede by far the $\mathrm{H} \alpha$ emission. This trend would probe the degree of interaction which increases with the evolutionary status. However, the possibility of a very massive PN (with CNO cycle) and the occurrence of shocks (increasing the $[\mathrm{SII}]$ emission) cannot be discarded. We underline the fact that among the PNe with ISM interaction other morphologically and spectroscopically similar structures can be hidden such as HII regions, SNRs and ejecta of WR shell. In those cases a multiwavelength identification appears to be the best solution for a proper discrimination.

\section{Conclusion}

Taking advantage of the depth of the IPHAS survey we were able to identify seven new PNe with ISM interaction ranging from the early to more advanced stages of interaction. The small sample indicates that the most evolved stages display greater $[\mathrm{NII}] / \mathrm{H} \alpha$ ratio at the interacting rim probing the occurrence of stronger interaction processes. In some cases we also probe the density of the local ISM which aims at being better established with a larger number of investigated PNe-ISM . More candidates have been discovered along the plane (particularly those with a one-sided morphology) and are awaiting spectroscopic identification and/or analysis.

\section{Acknowledgements}

LS is supported by PAPIIT-UNAM grant IN109509 (Mexico).

\section{References}

Ali, A., El-Nawawy, M. S., \& Pfleiderer, J. 2000, APSS, 271, 245

Borkowski, K. J., Sarazin, C. L., \& Soker, N. 1990, APJ, 360, 173

Drew, J. E., Greimel, R., Irwin, M. J., et al. 2005, MNRAS, 362, 753

Frew, D. J. \& Parker, Q. A. 2010, PASA, 27, 129

Sabin, L., Zijlstra, A. A., Wareing, C., et al. 2010, PASA, 27, 166

Wareing, C. J., Zijlstra, A. A., \& O'Brien, T. J. 2007, MNRAS, 382, 1233 